\title{
Soft Fermi Surfaces and Breakdown of Fermi Liquid Behavior
}

\author{
W. Metzner, D. Rohe, and S. Andergassen \\ Max-Planck-Institut für Festkörperforschung, D-70569 Stuttgart, Germany
}

November 4, 2018

\begin{abstract}
Electron-electron interactions can induce Fermi surface deformations which break the point-group symmetry of the lattice structure of the system. In the vicinity of such a "Pomeranchuk instability" the Fermi surface is easily deformed by anisotropic perturbations, and exhibits enhanced collective fluctuations. We show that critical Fermi surface fluctuations near a d-wave Pomeranchuk instability in two dimensions lead to large anisotropic decay rates for single-particle excitations, which destroy Fermi liquid behavior over the whole surface except at the Brillouin zone diagonal. PACS: 71.10.Fd, 74.20.Mn
\end{abstract}

Recently a number of authors pointed out that the Coulomb interaction between electrons in a metal can lead to Fermi surface deformations which break the orientational symmetry of the system. Referring to a stability criterion for normal Fermi liquids by Pomeranchuk, [1] we use the term "Pomeranchuk instability" (PI) for such symmetrybreaking Fermi surface shifts. Deformations of $d_{x^{2}-y^{2}}$-type, for which the Fermi surface expands along the $k_{x}$-axis and shrinks along the $k_{y}$-axis (or vice versa), have been found for various model Hamiltonians on a two-dimensional square lattice: t-J, 2] Hubbard, [3, 4, 5] and extended Hubbard model. [6] For a Fermi level close to the van Hove singularity the PI 
occurs already in the weak-coupling regime, where perturbative methods may be applied. Symmetry-broken Fermi surfaces in fully isotropic (not lattice) two- and three-dimensional Fermi liquids have also been considered. [7. From a pure symmetry-group point of view a PI leads to a "nematic" electron liquid as defined by Kivelson et al. [8] in their discussion of a possible correspondence between electron states in doped Mott insulators and liquid crystal phases.

Symmetry-breaking Fermi surface deformations generally compete with other instabilities, but may also coexist with other types of symmetry-breaking order. For example, a superconducting state with a d-wave deformed Fermi surface [9] is stabilized in the twodimensional Hubbard model with a sizable next-to-nearest neighbor hopping amplitude and an electron density near van Hove filling, at least at weak coupling. [5] Superconducting nematic states have also been considered as one among several possibilities in a general symmetry classification by Vojta et al. [10] In the following we will however focus on symmetry-breaking Fermi surface deformations in an otherwise normal state.

For electrons on a lattice, the PI breaks only a discrete symmetry, the point-group symmetry of the lattice. Hence, no Goldstone mode exists and symmetry-broken states can exist also at finite temperature in $d \geq 2$ dimensions. Order parameter fluctuations thus suppress the PI much less than competing instabilities toward states which would break a continuous symmetry. In the ground state a PI can be driven as a function of the electron density or other control parameters, leading to a quantum critical point at the transition between the symmetric and symmetry-broken states, provided that no first order transition occurs.

An interacting electron system in the vicinity of a PI is characterized by a "soft" Fermi surface, which can be deformed very easily, that is at low energy cost. In this article we show that strong dynamical fluctuations of such a soft Fermi surface affect physical properties of the system very strongly, and lead in particular to a very fast decay of single-particle excitations. The anisotropy of the Fermi surface fluctuations in a lattice 
system leads to a pronounced anisotropy of the single-particle decay rate. For $d_{x^{2}-y^{2}}$-wave fluctuations the decay is maximal near the $k_{x^{-}}$and $k_{y}$-axes and minimal near the diagonal of the Brillouin zone.

The "softness" of a Fermi surface can be quantified by the Fermi surface susceptibility [3]

$$
\kappa_{\mathbf{k}_{F} \mathbf{k}_{F}^{\prime}}=\frac{\delta s_{\mathbf{k}_{F}}}{\delta \mu_{\mathbf{k}_{F}^{\prime}}}
$$

which measures the Fermi surface shifts $\delta s_{\mathbf{k}_{F}}$ for small momentum dependent shifts of the chemical potential $\delta \mu_{\mathbf{k}_{F}^{\prime}}$ at points $\mathbf{k}_{F}^{\prime}$ on the Fermi surface. Close to a PI one of the eigenvalues of the matrix $\kappa_{\mathbf{k}_{F} \mathbf{k}_{F}^{\prime}}$ diverges, and the corresponding eigenvector describes the shape of the incipient Fermi surface deformation.

It is intuitively plausible that critical Fermi surface fluctuations will strongly affect single-particle excitations and as a consequence the low energy properties of the system. To explore the resulting physics, we define and analyze a phenomenological lattice model with an effective interaction chosen such that a PI occurs, but no other instabilities. The model Hamiltonian reads

$$
H=\sum_{\mathbf{k}, \sigma} \epsilon_{\mathbf{k}} n_{\mathbf{k} \sigma}+\frac{1}{2 V} \sum_{\mathbf{k}, \mathbf{k}^{\prime}, \mathbf{q}} f_{\mathbf{k k}^{\prime}}(\mathbf{q}) n_{\mathbf{k}}(\mathbf{q}) n_{\mathbf{k}^{\prime}}(-\mathbf{q})
$$

where $\epsilon_{\mathbf{k}}$ is a single-particle dispersion, $n_{\mathbf{k}}(\mathbf{q})=\sum_{\sigma} c_{\mathbf{k}-\mathbf{q} / 2, \sigma}^{\dagger} c_{\mathbf{k}+\mathbf{q} / 2, \sigma}$, and $V$ the volume of the system. Since the PI is driven by interactions with small momentum transfers, that is forward scattering, we choose a coupling function $f_{\mathbf{k k}^{\prime}}(\mathbf{q})$ which contributes only for relatively small momenta $\mathbf{q}$. This suppresses other instabilities such as superconductivity or density waves. We emphasize that this model is adequate only if Fermi surface fluctuations are the dominant fluctuations in the system. Otherwise it would have to be supplemented by other interactions with large momentum transfers. The interplay of Fermi surface and other fluctuations opens a wide field for investigations in the future.

For an analytical treatment we assume that the momentum dependence of the coupling 
function in (2) is separable, that is

$$
f_{\mathbf{k k}^{\prime}}(\mathbf{q})=g(\mathbf{q}) d_{\mathbf{k}} d_{\mathbf{k}^{\prime}}
$$

Although the above model can be defined in any dimension, we now focus on the particularly interesting case of a two-dimensional system on a square lattice. To generate a PI with $d_{x^{2}-y^{2}}$ symmetry, the form factors $d_{\mathbf{k}}$ must have that symmetry, such as $d_{\mathbf{k}}=\cos k_{x}-\cos k_{y}$, and $g(\mathbf{q})$ has to be negative, at least for $\mathbf{q} \rightarrow \mathbf{0}$. The resulting Landau function $f_{\mathbf{k k}^{\prime}}=f_{\mathbf{k k}^{\prime}}(\mathbf{0})$ captures qualitatively the most pronounced features of the Landau function obtained from renormalization group calculations [3] and perturbation theory [1] for the two-dimensional Hubbard model near van Hove filling. In particular, it is repulsive for momenta near two different van Hove points and attractive for momenta near a common one.

We compute the two-particle vertex function $\Gamma$ from $f$ by summing the series of bubble chains sketched in Fig. 1. By virtue of the separable structure of the interaction, the series can be summed algebraically yielding

$$
\Gamma_{\mathbf{k k}^{\prime}}(\mathbf{q}, \omega)=\frac{g(\mathbf{q})}{1-g(\mathbf{q}) \Pi_{d}(\mathbf{q}, \omega)} d_{\mathbf{k}} d_{\mathbf{k}^{\prime}},
$$

where $\Pi_{d}$ is the particle-hole bubble with a form factor $d_{\mathbf{k}}$ at the vertices, that is

$$
\Pi_{d}(\mathbf{q}, \omega)=-\int \frac{d^{2} p}{(2 \pi)^{2}} \frac{f\left(\epsilon_{\mathbf{p}+\mathbf{q} / 2}\right)-f\left(\epsilon_{\mathbf{p}-\mathbf{q} / 2}\right)}{\omega-\left(\epsilon_{\mathbf{p}+\mathbf{q} / 2}-\epsilon_{\mathbf{p}-\mathbf{q} / 2}\right)+i 0^{+}} d_{\mathbf{p}}^{2} .
$$

Here $f$ is the Fermi function. For small $\mathbf{q}$ and $\omega$ the d-wave bubble has the following asymptotic behavior: $\Pi_{d}(\mathbf{q}, 0)=-N_{d}^{F}+a(\hat{\mathbf{q}}) q^{2}+\mathcal{O}\left(q^{4}\right)$ and $\operatorname{Im}_{d}(\mathbf{q}, \omega) \rightarrow-c(\hat{\mathbf{q}}) \omega / q$ for $q, \omega \rightarrow 0$ and $\omega / q \rightarrow 0$, where $N_{d}^{F}>0$ is a weighted density of states, with each state at the Fermi level weighted by the squared form factor $d_{\mathbf{k}_{F}}^{2}$, while $a(\hat{\mathbf{q}})$ and $c(\hat{\mathbf{q}})$ are real coefficients depending only on the direction $\hat{\mathbf{q}}$ of $\mathbf{q}$, but not on its length. The coefficient $c(\hat{\mathbf{q}})$ is always positive, but the sign of $a(\hat{\mathbf{q}})$ depends on $\hat{\mathbf{q}}$ and on the choice of $\epsilon_{\mathbf{k}}, d_{\mathbf{k}}$ and $\mu$. 
The PI sets in when the denominator of Eq. (4) vanishes at zero frequency and vanishing momenta, that is $\lim _{\mathbf{q} \rightarrow 0} g(\mathbf{q}) \Pi_{d}(\mathbf{q}, 0)=-g(\mathbf{0}) N_{d}^{F}=1$ at the critical point. We assume that the $\mathbf{q}$-dependence of $g(\mathbf{q})$ is such that $g(\mathbf{q}) \Pi_{d}(\mathbf{q}, 0)<1$ for $\mathbf{q} \neq 0$ at that point. Otherwise an instability with a finite q-vector would set in first. For a small q-vector this would lead to a phase where the symmetry-breaking Fermi surface deformations are slowly modulated across the system. We leave this case for future studies.

At the critical point the two-particle vertex has the following asymptotic form for small $\mathbf{q}, \omega$ and small $\omega / q$ :

$$
\Gamma_{\mathbf{k k}^{\prime}}(\mathbf{q}, \omega) \sim \frac{d_{\mathbf{k}} d_{\mathbf{k}^{\prime}}}{i c(\hat{\mathbf{q}}) \omega / q-\alpha(\hat{\mathbf{q}}) q^{2}}
$$

with $\alpha(\hat{\mathbf{q}})=a(\hat{\mathbf{q}})-N_{d}^{F} g^{\prime \prime}(\hat{\mathbf{q}}) / g(\mathbf{0})$, where $g^{\prime \prime}(\hat{\mathbf{q}})$ is the second derivative of $g(\mathbf{q})$ with respect to $q$ for $q \rightarrow 0$. The denominator of the vertex has the same form as for other familiar critical points in metals, namely at the boundary to ferromagnetic [12, 13] or phase separated [14] states. Peculiar to the d-wave PI are the form factors in the numerator.

We stress that the PI obeys the symmetry conditions for a continuous phase transition, since odd powers of the order parameter are excluded by symmetry from the corresponding Landau theory, in contrast to the case of phase separation, where cubic terms usually drive a first order transition (except for special cases where the cubic term is tuned to zero). Note also that the Pomeranchuk instability is not inhibited by long-range Coulomb forces, since volume conserving Fermi surface deformations do not generate charge inhomogeneities.

To estimate the decay rate for single-particle excitations in the presence of critical Fermi surface fluctuations near the quantum critical point, we have computed the imaginary part of the self-energy to first order in $\Gamma_{\mathbf{k k}^{\prime}}(\mathbf{q}, \omega)$, evaluating the Feynman diagram in Fig. 2. For momenta on the Fermi surface, the result is

$$
\operatorname{Im} \Sigma\left(\mathbf{k}_{F}, \epsilon\right) \propto d_{\mathbf{k}_{F}}^{2} \epsilon^{2 / 3}
$$


for small energies $\epsilon$ at $T=0$ and

$$
\operatorname{Im} \Sigma\left(\mathbf{k}_{F}, 0\right) \propto d_{\mathbf{k}_{F}}^{2} T^{2 / 3}
$$

at low finite temperatures. Note that the integral leading to the above result is dominated by small momentum transfers $\mathbf{q}$ and $\omega \ll v_{\mathbf{k}_{F}} q$, thus justifying the asymptotic expansion of the vertex function. Furthermore, the dominant contributions to $\operatorname{Im} \Sigma$ at the Fermi vector $\mathbf{k}_{F}$ come from (small) $\mathbf{q}$-vectors that are tangential to the Fermi surface at $\mathbf{k}_{F}$.

Not unexpectedly, the decay rate has the same energy dependence as for the quantum critical point near phase separation in two dimensions. 14] Different is however the d-wave form factor making the decay rate strongly anisotropic. The decay rate is strongest near the van Hove points, while the leading terms vanish on the diagonal of the Brillouin zone. Subleading terms will produce at least conventional Fermi liquid decay rates $\left(T^{2} \log T\right)$ on the diagonal, but faster decay (intermediate between $T^{2 / 3}$ and $T^{2} \log T$ ) may be obtained due to higher order processes and interactions with large momentum transfers, which couple different parts of the Fermi surface.

The singular self-energy in the critical region will drastically modify the one-particle propagator at low energies. However, this does not invalidate the calculation of the twoparticle vertex from bubbles with bare propagators, since singular self-energy corrections generated by strong forward scattering are cancelled by corresponding vertex corrections in the polarization bubble.[15] A more subtle point is whether higher order corrections to the self-energy contribution in Fig. 2 modify the power-laws (7) and (8) for the decay rate. The same question has been discussed at length for fermions coupled to a gauge field, 15] where the lowest order calculation also yields a power-law with exponent 2/3. 16] A detailed analysis by Altshuler et al. [17] indicated that this result is not changed by other terms. A recent renormalization group analysis further supported the validity of the leading power-law for the gauge theory, and also for the critical point near phase separation in two dimensions. [18] Even if the above power-law would be modified by 
higher order corrections, it is clear that Fermi liquid behavior cannot be restored, and the decay rate will remain large and anisotropic in any case.

A strongly anisotropic decay rate following a power-law with exponent $2 / 3$ has recently been derived for an isotropic continuum (not lattice) version of model (2). [7] That result was obtained for the symmetry-broken "nematic" phase, and the anisotropy of the decay rate arises from the anisotropy of the symmetry-broken state and its collective modes. In the symmetric phase of an isotropic liquid the decay rates are of course isotropic. At the quantum critical point the decay rate of the isotropic liquid also obeys a power law with exponent $2 / 3$, but now isotropically over the whole Fermi surface.

Could soft Fermi surfaces and critical Fermi surface fluctuations play a role in cuprate superconductors? Due to the coupling of electron and lattice degrees of freedom a symmetry-breaking Fermi surface deformation is generally accompanied by a lattice distortion, and vice versa. Structural transitions which reduce the lattice symmetry of the cuprate-planes are quite frequent in cuprates. Close to a PI of the electronic system, electronic properties can be expected to react unusually strongly to slight lattice distortions. Such "overreactions" of electronic properties have indeed been observed in several cuprate compounds. 19] In particular, a slight orthorhombicity of the lattice structure would lead to a relatively strong orthorhombic distortion of the Fermi surface. Yamase and Kohno [20] invoked this idea to explain peculiarities of magnetic excitations in cuprates.

Large Fermi surface fluctuations could be at least partially responsible for the nonFermi liquid behavior observed in cuprates at optimal doping. In our model calculation we have obtained a strongly anisotropic anomalously large decay rate for single-particle excitations. Large anisotropic decay rates have been frequently inferred from the linewidth observed in photoemission experiments on optimally doped cuprates. 21] However, recent experiments with higher resolution revealed that bilayer splitting of the bands has to be taken into account in the data analysis, such that the intrinsic linewidth near the van Hove points may be considerably smaller and less anisotropic than previously expected. [22] 
As to the temperature dependence of the decay rate, it is presently hard to reliably discriminate a $T$-linear from a $T^{2 / 3}$ behavior of the intrinsic linewidth extracted from the experimental data. Concerning transport, an anisotropic scattering rate with nodes on the diagonal can very naturally account for the pronounced anisotropy between intraand inter-plane mobility of charge carriers, as pointed out by Ioffe and Millis [23] in their phenomenological "cold spot" scenario. According to their idea, the intra-plane transport is dominated by quasi-particles with a long life-time near the diagonal of the Brillouin zone, while these carriers are not available for inter-plane transport, since transverse hopping amplitudes vanish on the diagonal. To analyze transport properties near a PI one has to face the notorious difficulties of transport theory in a quantum critical regime. Even for rough estimates of temperature dependences within a semiclassical Boltzmann approach one would have to compute subleading corrections to the decay rates, since those limit the life-time of excitations on the Brillouin zone diagonal, and thus the inplane conductivities. Furthermore, regular interactions or interactions peaked at finite q-vectors (leading to "hot spots" on the Fermi surface) should also play a role, and may lead to an interesting but complicated interplay with Fermi surface fluctuations.

Since the PI breaks the orientational symmetry of the lattice, it is natural to consider a possible connection with the tendency towards stripe formation, which has been extensively discussed in the context of cuprate superconductors. 24. Stripes also break the translation invariance in addition to orientational symmetry, and their formation requires interactions with large momentum transfers, such as antiferromagnetic interactions. The possibility of direct transitions between states with broken orientational order and stripe states, which has been envisaged already by Kivelson et al. [8], is an interesting subject for future studies.

In summary, an electron system close to a PI is characterized by a soft Fermi surface, which can react strongly to a slight change of the lattice structure, and exhibits strong collective Fermi surface fluctuations. In two-dimensional systems these fluctu- 
ations lead to large anisotropic decay rates for single-particle excitations and thus to a breakdown of Fermi liquid theory. It will be interesting to further explore the consequences of Pomeranchuk criticality, especially for charge and heat transport, for magnetism, and for superconductivity.

\section{Acknowledgements:}

We are very grateful to Claudio Castellani for contributing several valuable ideas and insights, especially at the initial stage of this work. We also thank B. Büchner, B. Keimer, C. Di Castro, G. Khaliullin, G. Kotliar, V. Oganesyan, M. Salmhofer, F. Wegner, H. Yamase, and R. Zeyher for useful discussions. This work was initiated at the workshop "Realistic theories of correlated electron materials" in fall 2002 at ITP-UCSB, and supported by the DFG-grant Me 1255/6-1.

\section{References}

[1] I.J. Pomeranchuk, Sov. Phys. JETP 8, 361 (1958).

[2] H. Yamase and H. Kohno, J. Phys. Soc. Jpn. 69, 332 (2000); 69, 2151 (2000).

[3] C.J. Halboth and W. Metzner, Phys. Rev. Lett. 85, 5162 (2000).

[4] I. Grote, E. Körding, and F. Wegner, J. Low Temp. Phys. 126, 1385 (2002); V. Hankevych, I. Grote, and F. Wegner, Phys. Rev. B 66, 094516 (2002).

[5] A. Neumayr and W. Metzner, Phys. Rev. B 67, 035112 (2003).

[6] B. Valenzuela and M.A.H. Vozmediano, Phys. Rev. B 63153103 (2001).

[7] V. Oganesyan, S.A. Kivelson, and E. Fradkin, Phys. Rev. B 64, 195109 (2001).

[8] S.A. Kivelson, E. Fradkin, and V.J. Emery, Nature 393, 550 (1998). 
[9] Although the discontinuity of the momentum distribution function is smeared by the gap in a superconductor, a Fermi surface can still be defined as the locus of zeros of the diagonal components of the inverse Nambu propagator in momentum space.

[10] M. Vojta, Y. Zhang, and S. Sachdev, Phys. Rev. Lett. 85, 4940 (2000); Int. J. Mod. Phys. B 14, 3719 (2000).

[11] P.A. Frigeri, C. Honerkamp, and T.M. Rice, Eur. Phys. J. B 28, 61 (2002).

[12] J.A. Hertz, Phys. Rev. B 14, 1165 (1976).

[13] A.J. Millis, Phys. Rev. B 48, 7183 (1993).

[14] C. Castellani, C. Di Castro, and M. Grilli, Phys. Rev. Lett. 75, 4650 (1995).

[15] For a review on Fermi systems with strong forward scattering, see W. Metzner, C. Castellani, and C. Di Castro, Adv. Phys. 47, 317 (1998).

[16] P.A. Lee, Phys. Rev. Lett. 63, 680 (1989); B. Blok and H. Monien, Phys. Rev. B 47, 3454 (1993).

[17] B.L. Altshuler, L.B. Ioffe, and A.J. Millis, Phys. Rev. B 50, 14048 (1994).

[18] C. Castellani, S. Caprara, C. Di Castro, and A. Maccarone, Nucl. Phys. B 594, 747 (2001).

[19] J.D. Axe et al., Phys. Rev. Lett. 62, 2751 (1989); B. Büchner et al., Phys. Rev. Lett. 73, 1841 (1994).

[20] H. Yamase and H. Kohno, J. Phys. Soc. Jpn. 70, 2733 (2001); ibid 71, 1154 (2002).

[21] See, for example, T. Valla et al., Phys. Rev. Lett. 85, 828 (2000).

[22] For a recent review on photoemission in cuprates, see A. Damascelli, Z. Hussain, and Z.X. Shen, Rev. Mod. Phys. 75, 473 (2003). 
[23] L.B. Ioffe, and A.J. Millis, Phys. Rev. B 58, 11631 (1998).

[24] See, for example, Proc. of 3rd Int. Conf. on Stripes and High $T_{c}$ superconductivity, Int. J. Mod. Phys. B 14, Nos. 29-31 (2000). 


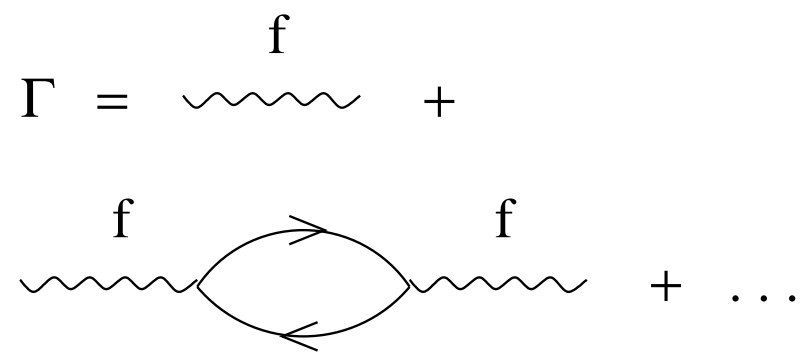

Figure 1: Series of bubble chains contributing to the two-particle vertex $\Gamma$.

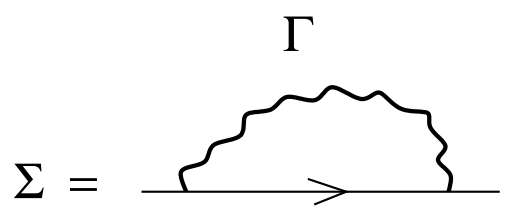

Figure 2: Feynman diagram relating the self-energy $\Sigma$ to the two-particle vertex $\Gamma$. 